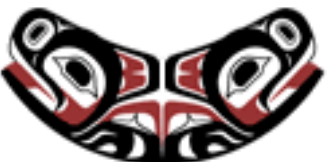

UW Biostatistics Working Paper Series

6-13-2003

\title{
Tests for Comparing Mark-Specific Hazards and Cumulative Incidence Functions
}

Peter B. Gilbert

Fred Hutchinson Cancer Research Center \& University of Washington, pgilbert@scharp.org

Ian W. McKeague

Florida State University,im2131@columbia.edu

Yanqing Sun

University of North Carolina at Charlotte, yasun@uncc.edu

\section{Suggested Citation}

Gilbert, Peter B.; McKeague, Ian W.; and Sun, Yanqing, "Tests for Comparing Mark-Specific Hazards and Cumulative Incidence Functions" (June 2003). UW Biostatistics Working Paper Series. Working Paper 209.

http://biostats.bepress.com/uwbiostat/paper209

This working paper is hosted by The Berkeley Electronic Press (bepress) and may not be commercially reproduced without the permission of the copyright holder.

Copyright $(\odot) 2011$ by the authors 


\section{Introduction}

Many studies of survival data involve mark variables that are only observed at an endpoint event and it is of interest to investigate whether there is any relationship between the time to endpoint and the mark variable. For example, in a clinical trial of drug regimens for treating HIV infection, the time to treatment failure (typically defined by levels of viral load rising above a threshold (Gilbert et al., 2001)) can decrease with increases in a distance measure describing the extent of drug-selected HIV genetic evolution within a patient between baseline and the time of failure. Detecting such an association can help in designing anti-HIV treatments that overcome the problem of drug resistance, which represents one of the greatest barriers to achieving durably efficacious treatment of HIV infection (Hirsch et al. 2000; Yeni et al. 2002).

In this article we develop tests for detecting whether a mark-specific hazard rate (or cumulative incidence function) depends on the mark, and apply the tests to HIV genetic data collected in an AIDS clinical trial. If we denote the time to endpoint $T$ and the mark variable $V$, the observable random variables are $(X, \delta, \delta V)$, where $X=\min \{T, C\}, \delta=I(T \leq C)$, and $C$ is a censoring random variable that is assumed to be independent of $T$ and $V$. When the failure time $T$ is observed, $\delta=1$ and the mark $V$ is also observed, whereas if $T$ is censored, the mark is unknown. Statistical interest focuses on the mark-specific hazard rate function

$$
\lambda(t, v)=\lim _{h_{1}, h_{2} \rightarrow 0} P\left\{T \in\left[t, t+h_{1}\right), V \in\left[v, v+h_{2}\right) \mid T \geq t\right\} / h_{1} h_{2},
$$

and the cumulative incidence function

$$
F(t, v)=\lim _{h_{2} \rightarrow 0} P\left\{T \leq t, V \in\left[v, v+h_{2}\right)\right\} / h_{2},
$$

with $t$ ranging over a fixed interval [0, $\tau]$. If $V$ is discrete, the limit $h_{2} \rightarrow 0$ is not needed, and the definitions (1) and (2) simplify respectively to the discrete cause-specific hazard function and the discrete cumulative incidence function, which have received much attention in the competing risks literature. In this article, the mark variable $V$ is assumed to be continuous, in which case the functions (1) and (2) are the natural analogs of their discrete counterparts, with similar interpretations. In particular, $\lambda(t, v)$ is the instantaneous risk of failure by a cause $V$ in a small interval $\left[v, v+h_{2}\right)$ in the presence of all other causes, and $F(t, v)$ is the probability that failure with $V$ in a small interval $\left[v, v+h_{2}\right)$ will occur before the specified time $t$.

As with the classic competing risks model, the mark-specific hazard relates the cumulative incidence function through the simple formula $F(t, v)=\int_{0}^{t} \lambda(s, v) S_{T}(s) d s$, where $S_{T}(t)$ is the 
survival function of $T$. Furthermore, just as the cause-specific hazard functions are the basic estimable quantities when the mark variable is discrete (as originally pointed out by Prentice et al., 1978), the mark-specific hazard function (1) is estimable from the available data and forms the basis for inference when the mark variable is continuous. Indeed, the likelihood function under the competing risks data with continuous mark has a similar form and is derived as follows. Assume that the continuous mark variable $V$ has a known bounded support; rescaling $V$ if necessary, this support is taken to be $[0,1]$. Let $f(t, v)$ be the joint density of $(T, V)$. Then $\lambda(t, v)=f(t, v) / S_{T}(t)$ and $\lambda(t)=\int_{0}^{1} \lambda(t, v) d v$ is the overall hazard function of $T$. The likelihood function given $n$ i.i.d. observations $\left(X_{i}, \delta_{i}, \delta_{i} V_{i}\right), i=1, \ldots, n$ from the above model can be expressed in terms of the mark-specific hazard rate as

$$
\prod_{o} f\left(X_{i}, V_{i}\right) \prod_{c} S_{T}\left(X_{i}\right)=\prod_{o} \lambda\left(X_{i}, V_{i}\right) \prod_{i=1}^{n} \exp \left\{-\int_{0}^{1} \int_{0}^{X_{i}} \lambda(s, v) d s d v\right\},
$$

where $\prod_{o}$ denotes the product over the observed failure times, $\prod_{c}$ denotes the product over right censored failure times, and each product only applies to the expression immediately in front. These considerations motivate us to develop an inferential procedure based on the function (1).

Our interest centers on testing the null hypothesis

$$
H_{0}: \lambda(t, v) \text { does not depend on } v \text { for } t \in[0, \tau]
$$

against the following alternative hypotheses:

$$
\begin{aligned}
& H_{1}: F\left(t, v_{1}\right) \leq F\left(t, v_{2}\right) \text { for all } v_{1} \leq v_{2}, t \in[0, \tau] \\
& H_{2}: \lambda\left(t, v_{1}\right) \leq \lambda\left(t, v_{2}\right) \text { for all } v_{1} \leq v_{2}, t \in[0, \tau] \\
& H_{3}: \lambda\left(t, v_{1}\right) \neq \lambda\left(t, v_{2}\right) \text { for some } v_{1} \leq v_{2}, t \in[0, \tau]
\end{aligned}
$$

with strict inequalities for some $t, v_{1}, v_{2}$ in both $H_{1}$ and $H_{2}$. Equivalently, $H_{0}$ can be expressed in terms of the cumulative incidence function $F(t, v)$ not depending on $v$ for $t \in[0, \tau]$. The null hypothesis $H_{0}$ can also be written as $H_{0}: \lambda(t, v)=\lambda(t)$ for all $t \in[0, \tau]$ and $v \in[0,1]$. Expressed in this way, $H_{0}$ is the continuous version of the null hypothesis considered by Aly, Kochar, and McKeague (1994), who developed a test for equality of two discrete cause-specific hazard rates, $H_{0}: \lambda_{1}(t)=\lambda_{2}(t)$ for all $t \in[0, \tau]$.

As for the case of discrete competing risks, the interpretation of inferences on the mark-specific hazard function $\lambda(t, v)$ is restricted to actual study conditions (i.e., is "crude" or "gross"), and there is no implication that the same inference would be made under a new set of conditions in 
which, for example, certain causes of failure $v$ were not present. With $T_{v}$ denoting the latent (i.e., notional) failure time for mark $v$ (see Prentice et al., 1978, for discussion of latent failure times), the assumption of mutual independence of the $T_{v}$ for all $v \in[0,1]$ is needed for $\lambda(t, v)$ to possess the stronger interpretation as the hazard function for cause $v$ given that all other causes are inoperative. As in the discrete case, the independence assumption is untestable from the available competing risks data (cf., Tsiatis, 1975); additional data such as observations of marks beyond the first failure time are needed. Thus, tests of $H_{0}$ have an interpretation in terms of association, and cannot be used for causal inference of the predictive effect of a mark variable on the risk of failure. The fact that the mark variable is only observed simultaneously with failure makes clear the impossibility of causal inference (in the absence of strong untestable assumptions).

In the AIDS clinical trial example, $V$ is a measure of the accumulated HIV genetic resistance resulting from exposure to an antiretroviral treatment, which is measured only on subjects who fail treatment, at the time of treatment failure. The test of $H_{0}$ versus the monotone alternative $H_{1}\left(H_{2}\right)$ assesses whether the absolute (instantaneous) risk of treatment failure increases with the level of acquired drug resistance. If $V$ is a reliable measure of the "resistance cost" of the regimen, i.e., if the risk of treatment failure is higher for larger values of $V$, then we would expect to reject $H_{0}$ in favor of $H_{2}$. Thus, the test is useful for evaluating if $V$ is a clinically relevant measure of a treatment's resistance cost (see Gilbert et al. 2000 for a discussion of relevant resistance cost metrics). Knowledge of clinically meaningful genetic resistance cost metrics would be helpful for identifying combination drug regimens that do not select for drug resistant virus, and thus provide long-lasting treatment efficacy.

A second example in which the proposed approach would be of interest is a prospective cohort study of a population at risk for acquiring HIV infection. In this application, $T$ is the time from cohort entry until HIV infection, and $V$ is the value of a metric measuring genotypic or phenotypic dissimilarity of the HIV virus that infects a study participant from a reference HIV strain. For example, $V$ could be Hamming's genetic distance and the reference strain could be the prototype virus contained in an HIV vaccine that is under development for field testing in the cohort population. The test of $H_{0}$ versus the two-sided hypothesis $H_{3}$ assesses whether the HIV metric $V$ is associated with the instantaneous risk of HIV infection. Finding evidence for $H_{3}$ may suggest that the metric $V$ can be used to guide selection of the types of HIV antigens to include in HIV vaccines (Gilbert et al., 2001). For example, if $H_{0}$ is rejected and the infection risk appears particularly high for $v>0.7$, then it may behoove vaccine researchers to insert HIV 
antigens characterized by $v>0.7$. Carrying out the test for multiple metrics in multiple genes could help identify the metric(s) that optimize the breadth of expected protective coverage of the vaccine. This application is important because the broad genotypic and phenotypic diversity of HIV poses one of the greatest challenges to developing an effective AIDS vaccine (UNAIDS, 2001).

In the case of a discrete mark variable, tests for comparing mark-specific hazards can be found in the literature on competing risks, see, e.g., Aly, Kochar, and McKeague (1994), Sun and Tiwari (1995), Lam (1998), Hu and Tsai (1999), Luo and Turnbull (1999) and Sun (2001). Fine (1999) developed a semiparametric regression method for competing risks data in which a discrete mark $V$ was used to stratify the effects of other covariates. This approach could not be used directly for continuous marks because the subgroup with a given mark $V=v$ would be empty or contain one subject. Nevertheless, this work suggests that one may explicitly model the effect of continuous mark $V$ on the cumulative incidence function or mark-specific hazard rate. As mentioned earlier, our testing procedure can be viewed as a continuous extension of the procedure of Aly, Kochar, and McKeague (1994) and Sun (2001). To the best of our knowledge, however, these tests have not been developed for continuous mark variables.

A nonparametric estimator of the joint distribution of a failure time and a failure mark which may be continuous has been introduced by Huang and Louis (1998), with a view to applications such as evaluating the relationship between a quality of life score and survival time (Olschewski and Schumacher, 1990), or between lifetime medical cost and survival time. Their estimator could be used to test whether $T$ and $V$ are independent by comparing it with the product of its marginals. A test statistic based on this approach would have a complex asymptotic distribution, however, and it is not clear that a tractable testing procedure could be formulated. Furthermore, given the interpretability of the mark-specific hazard function in terms of the instantaneous risk of failure, we argue that in some biomedical problems testing $\lambda(t, v)$ independent of $v$ is more directly relevant than testing $T$ and $V$ independent. For example, for an HIV infected patient receiving effective antiretroviral treatment at a given time, the risk of treatment failure over the next month is of primary clinical interest, and is measured by the hazard function; accordingly the relationship between the mark variable and the hazard function is of direct clinical interest.

In the case of finitely many causes of failure, test statistics can be based on differences between Nelson-Aalen estimators of the cumulative cause-specific hazard functions, see Sun (2001). Generalizing this approach, our test procedure is based on estimates of the doubly cumulative 
mark-specific hazard function $\Lambda(t, v)=\int_{0}^{v} \int_{0}^{t} \lambda(s, u) d s d u$; estimation of this function was also used in a fundamental way by Huang and Louis (1998). The idea of our testing procedure is to compare a nonparametric estimate of $\Lambda(t, v)$ with an estimate under $H_{0}$. We show that the comparison can be weighted to make the test statistics asymptotically distribution-free.

This article is organized as follows. In Section 2 we introduce the test statistics and describe a Monte Carlo procedure for approximating critical values. In Section 3 we derive the asymptotic null distributions of the test statistics, and show that the Monte Carlo-derived critical values are asymptotically accurate. The results of a simulation study and the AIDS clinical trial example are presented in Sections 4 and 5, and proofs of results are given in the Appendix.

\section{Test procedure}

Given observation of i.i.d. replicates $\left(X_{i}, \delta_{i}, \delta_{i} V_{i}\right), i=1, \ldots, n$ of the (possibly right-censored) marked failure times, a suitable nonparametric estimator of $\Lambda(t, v)$ is provided by the NelsonAalen-type estimator

$$
\hat{\Lambda}(t, v)=\int_{0}^{t} \frac{N(d s, v)}{Y(s)}, t \in[0, \tau], v \in[0,1],
$$

where $Y(t)=\sum_{i=1}^{n} I\left(X_{i} \geq t\right)$ is the size of the risk set at time $t$, and

$$
N(t, v)=\sum_{i=1}^{n} I\left(X_{i} \leq t, \delta_{i}=1, V_{i} \leq v\right)
$$

is the marked counting process with jumps at the uncensored failure times $X_{i}$ and associated marks $V_{i}$, cf. Huang and Louis (1998, eq. 3.2). From (3), the log-likelihood can be expressed as

$$
\int_{0}^{1} \int_{0}^{\tau} \log \lambda(s, v) N(d s, d v)-\int_{0}^{1} \int_{0}^{\tau} Y(s) \lambda(s, v) d s d v
$$

and it follows by a routine extension of the argument of Andersen et al. (1993, p. 228) that $\hat{\Lambda}$ is the nonparametric maximum likelihood estimator of $\Lambda$.

A closely related doubly cumulative hazard function estimator was introduced by McKeague and Utikal (1990) and McKeague, Nikabadze, and Sun (1995), for testing independence of a covariate from a failure time. The two estimators are not interchangeable, however, because the covariate and the mark variable play different roles in each setting. The doubly cumulative hazard function estimator of McKeague, Nikabadze, and Sun (1995) is formed by stratifying on the covariate, which cannot be done on the mark for competing risks data since the mark is not observed under censoring. The risk set at time $t$ for the competing risks data cannot be stratified by the marks since they are not available until the failures are observed. Although the 
current approach could be used to test for independence between a failure time and a covariate, it would be more appropriate to use the test of McKeague, Nikabadze, and Sun (1995), leading to a stronger conclusion when the null hypothesis is rejected. We also note here in passing that it is important to distinguish a mark variable from a "marker"; the latter term is synonymous for "covariate" in the survival analysis literature.

Because $H_{0}$ can be expressed as $H_{0}: \lambda(t, v)=\lambda(t)$ for all $t \in[0, \tau]$ and $v \in[0,1]$, and $\lambda(t, v)=f(t, v) / S_{T}(t)$, it follows that $H_{0}$ holds if and only if $f(t, v)=f_{T}(t)$ for all $t$ and $v$, where $f_{T}(t)$ is the density of $T$. Consequently, $H_{0}$ holds if and only if $T$ and $V$ are independent and $V$ is uniformly distributed over [0,1]. Because of this fact, we can write $\Lambda(t, v)=v \Lambda(t, 1)$, where $\Lambda(\cdot, 1)$ is the cumulative hazard function of $T$ under $H_{0}$. Thus, under $H_{0}$ we can estimate the doubly cumulative hazard function by $\bar{\Lambda}(t, v)=v \hat{\Lambda}(t, 1)$.

\subsection{Test processes and test statistics}

We consider test processes of the form

$$
L_{n}(t, v)=\sqrt{n} \int_{0}^{t} H_{n}(s)(\hat{\Lambda}-\bar{\Lambda})(d s, v)
$$

for $t \geq 0,0 \leq v \leq 1$, where $H_{n}(\cdot)$ is a suitable weight process. The weight process $H_{n}(\cdot)$ provides a flexible way to specify the relative importance attached to differences in the mark-specific hazards at different times, and is useful for controlling instability in the tails. The bivariate test process $L_{n}(t, v)$ is similar to the univariate test process $L_{n}(t)$ used by Aly, Kochar, and McKeague (1994, p. 996) for comparing two competing risks 1 and 2 , given by $L_{n}(t)=\int_{0}^{t} w(s) d\left(\hat{\Lambda}_{1}-\hat{\Lambda}_{2}\right)(s)$, with $\hat{\Lambda}_{j}(\cdot)$ the cause- $j$-specific Nelson-Aalen estimator.

Let $y(t)=P(X \geq t)$ and $\tilde{\tau}=\sup \{t: y(t)>0\}$ and assume $\tau<\tilde{\tau}$. We propose the following test statistics to measure departures from $H_{0}$ in the direction of $H_{1}, H_{2}$ and $H_{3}$ :

$$
\begin{gathered}
U_{1}=\sup _{v_{1}<v_{2}} \sup _{0 \leq t<\tau} \Delta\left(t, v_{1}, v_{2}\right) \\
U_{2}=\sup _{v_{1}<v_{2}} \sup _{0 \leq s \leq t<\tau}\left(\Delta\left(t, v_{1}, v_{2}\right)-\Delta\left(s, v_{1}, v_{2}\right)\right) \\
U_{3}=\sup _{v_{1}<v_{2}} \sup _{0 \leq t<\tau}\left|L_{n}\left(t, v_{1}\right)-L_{n}\left(t, v_{2}\right)\right|
\end{gathered}
$$

where $\Delta\left(t, v_{1}, v_{2}\right)=L_{n}\left(t, v_{1}\right)+L_{n}\left(t, v_{2}\right)-2 L_{n}\left(t,\left(v_{1}+v_{2}\right) / 2\right)$.

If the marks are discretized into $K$ groups by stratifying the marks into $K$ intervals of equal length, the proposed tests reduce to the tests of Aly, Kochar, and McKeague (1994) for $K=2$ and are equivalent to the tests developed by Sun (2001). However, the tests using discrete marks could 
have nearly zero power to detect certain alternatives when the underlying marks are continuous. This point will be discussed further following Theorem 3 on the omnibus property of the proposed tests.

In the next section we show that $L_{n}(t, v)$ converges weakly to a Gaussian process under $H_{0}$. We also show that the proposed tests based on the $U_{j}$ are consistent against their respective alternatives. Since each $U_{j}$ is a continuous functional of $L_{n}(t, v)$, its limiting null distribution is the distribution of the corresponding functional of the limiting Gaussian process. These distributions are intractable, however, so the critical values of the $U_{j}$ need to be determined using a simulation procedure.

\subsection{Monte Carlo procedure}

The procedure is based on a randomized version $U_{j}^{*}$ of $U_{j}$ defined by replacing each $V_{i}$ by $V_{i}^{*}$, where $V_{1}^{*}, \ldots, V_{n}^{*}$ are i.i.d. uniform $[0,1]$ random variables. This yields a randomized version of the test process given by

$$
L_{n}^{*}(t, v)=\sqrt{n} \int_{0}^{t} H_{n}(s)\left(\hat{\Lambda}^{*}(d s, v)-v \hat{\Lambda}^{*}(d s, 1)\right),
$$

where $\hat{\Lambda}^{*}(t, v)=\int_{0}^{t} N^{*}(d s, v) / Y(s)$ and $N^{*}(t, v)=\sum_{i=1}^{n} I\left(X_{i} \leq t, \delta_{i}=1, V_{i}^{*} \leq v\right)$. Exploiting the property that $T$ and $V$ are independent and $V$ is uniformly distributed over [0,1] under $H_{0}$, in Section 3 we show that the null distribution of $U_{j}$ coincides in the limit with the conditional distribution of $U_{j}^{*}$ given the observed data. Therefore a critical value of $U_{j}$ can be approximated via a Monte Carlo estimate of the quantile of $U_{j}^{*}$ corresponding to a given level of the test.

\subsection{Choice of weight process and a graphical procedure}

The simplest weight process, $H_{n}(t)=1$, yields a test process equal to a normalized difference of estimated doubly cumulative mark-specific hazard functions evaluated at $v$ and at 1 :

$$
L_{n}(t, v)=\sqrt{n}(\hat{\Lambda}(t, v)-v \hat{\Lambda}(t, 1))
$$

This process is useful for a graphical procedure, in which the surface $L_{n}(t, v)$ is plotted together with 10 or 20 realizations of the simulated null surface $L_{n}^{*}(t, v)$. Relative to the reference processes $L_{n}^{*}(t, v)$, large values of curvature of $L_{n}(t, v)$ in $v$ suggest $H_{1}$, an increasing trend of this curvature with time suggests $H_{2}$, and absolute differences in $L_{n}(t, v)$ over different mark values suggest $H_{3}$. The graphical procedure is illustrated in the example given in Section 5 . 
To give the tests maximal power, the weight process should be chosen to downweight the comparison of mark-specific hazards at larger times, where the test process is most variable. A weight process that accomplishes this is given by $H_{n}(t)=\hat{S}_{C}(t-) \hat{S}_{T}^{1 / 2}(t)$, where $\hat{S}_{C}$ and $\hat{S}_{T}$ are the Kaplan-Meier estimators of $S_{C}$ and $S_{T}$, respectively, $S_{C}$ being the survivor function of $C$. As shown in the next section, this weight process has the added advantage of making the test statistics asymptotically distribution-free.

\section{Large-sample results}

We begin by defining notation that is used in the sequel. Let $\gamma(t, v)=P(X \leq t, \delta=1, V \leq v)$. By the Glivenko-Cantelli Theorem, $N(t, v) / n$ and $Y(t) / n$ converge almost surely to $\gamma(t, v)$ and $y(t)$, uniformly in $(t, v) \in[0, \infty) \times[0,1]$ and $t \in[0, \infty)$, respectively. Let $D(I)$ be the Skorohod space for a $k$-dimensional rectangle $I$ (Bickel and Wichura 1971), and $C(I)$ be the subspace of continuous functions on $I$. Also, let $x \wedge y$ and $x \vee y$ denote the minimum and maximum of $x$ and $y$, respectively.

Our first result describes the limiting null distribution of the test process.

\section{Theorem 1}

Let the weight process $H_{n}(t)$ be a continuous functional of the processes $N(t, 1)$ and $Y(t), t \in[0, \tau]$, $\tau<\tilde{\tau}$. Assume there exists a measurable function $H(t)$ such that $\sup _{0 \leq t \leq \tau}\left|H_{n}(t)-H(t)\right| \stackrel{\text { a.s. }}{\longrightarrow}$ 0 and both $H_{n}$ and $H$ have bounded variation independent of $n$ almost surely. Then, under $H_{0}$

$$
L_{n}(t, v) \stackrel{\mathcal{D}}{\rightarrow} \int_{0}^{t} H(s) y(s)^{-1}\left(G_{1}(d s, v)-v G_{1}(d s, 1)\right) \equiv L(t, v)
$$

in $D([0, \tau] \times[0,1])$ as $n \rightarrow \infty$, where $G_{1}(t, v)$ and $G_{2}(t)$ are continuous mean zero Gaussian processes with covariances

$$
\begin{aligned}
\operatorname{Cov}\left(G_{1}(s, u), G_{1}(t, v)\right) & =\gamma(s \wedge t, u \wedge v)-\gamma(s, u) \gamma(t, v) \\
\operatorname{Cov}\left(G_{2}(s), G_{2}(t)\right) & =y(s \vee t)-y(s) y(t) \\
\operatorname{Cov}\left(G_{1}(t, v), G_{2}(s)\right) & =(\gamma(t, v)-\gamma(s-, v)) I(s \leq t)-\gamma(t, v) y(s) .
\end{aligned}
$$

The limiting process $L(t, v)$ is a mean zero Gaussian process with covariance

$$
\operatorname{Cov}(L(s, u), L(t, v))=(u \wedge v-u v) \int_{0}^{s \wedge t} \frac{H(r)^{2}}{y(r)^{2}} \gamma(d r, 1) .
$$

The process $L(t, v)$ resembles the Kiefer-Müller process (van der Vaart and Wellner, 1996, p. $226)$. 
We next establish that the randomized version of the test process $L_{n}^{*}(t, v)$, introduced in Section 2.2, has the same limiting null distribution as $L_{n}(t, v)$.

\section{Theorem 2}

Under the conditions of Theorem 1, conditional on the observed data sequence,

$$
L_{n}^{*}(t, v) \stackrel{\mathcal{D}}{\rightarrow} L(t, v)
$$

in $D([0, \tau] \times[0,1])$ under $H_{0}$, where $L(t, v)$ is given in Theorem 1 .

Theorem 2 justifies the Monte Carlo procedure described in Section 2.2, showing that it yields asymptotically correct critical values of the tests. Furthermore, under mild conditions the tests are consistent against their respective alternatives, as stated in Theorem 3.

\section{Theorem 3}

Suppose the conditions of Theorem 1 hold.

(a) If there exist $\left(t_{0}, v_{1}, v_{2}\right), 0<t_{0}<\tau,\left(v_{1}, v_{2}\right) \in[0,1]$, such that $H_{1}$ holds with strict inequality, $H(t) / S_{T}(t)$ is decreasing and $H\left(t_{0}\right)>0$, then the test based on $U_{1}$ is consistent against $H_{1}$.

(b) If there exist $\left(t_{0}, v_{1}, v_{2}\right), 0<t_{0}<\tau,\left(v_{1}, v_{2}\right) \in[0,1]$, such that $H_{2}$ holds with strict inequality, $H(t)$ and $\int_{0}^{v} \lambda(t, u) d u$ are continuous in $t$ in a neighborhood of $t_{0}$, and $H\left(t_{0}\right)>0$, then the test based on $\mathrm{U}_{2}$ is consistent against $\mathrm{H}_{2}$.

(c) If $\lambda(t, v)$ and $\lambda(t)$ are continuous on $[0, \tau] \times[0,1]$ and $[0, \tau]$, respectively, and $H(t) \geq c>0$ on $[0, \tau]$, then the test based on $U_{3}$ is consistent against $H_{3}$.

In practice, one may conveniently discretize the marks and apply the tests in Aly, Kochar, and McKeague (1994) and Sun (2001). We caution that such procedures could have nearly zero power to detect certain alternatives for the underlying continuous marks. For instance, if the marks are grouped into two categories with $V=0.25$ for those in the interval $(0,0.5)$ and $V=0.75$ for those in the interval $(0.5,1)$, then the test statistics $U_{1}, U_{2}$ and $U_{3}$ reduce to the tests of Aly, Kochar, and McKeague (1994). Let $H_{n}(t)=1$ and choose a model with continuous mark such that $\int_{0.25}^{0.75} \lambda(t, u) d u-0.5 \lambda(t)=0$ for all $t$, but $\lambda(t, v) \neq \lambda(t)$. This equation is equivalent to $\int_{0.25}^{0.75} f(t, u) d u-0.5 f_{T}(t)=0$ for all $t$, but $f(t, v) \neq f_{T}(t)$. A simple example of such alternative is that $f(t, v)=f_{T}(t) f_{V}(v)$ with $f_{V}(v)=1-\delta+4 \delta(v-0.5)$ for $v \in(0.5,1)$ and $f_{V}(v)=1-\delta-4 \delta(v-0.5)$ for $v \in(0,0.5)$, where $0 \leq \delta \leq 1$. This alternative approaches the null hypothesis as $\delta \rightarrow 0$. It follows from the proof of Lemma 1 that $\gamma(d s, v)-v \gamma(d s, 1)=$ 
$P(C \geq s)\left[\int_{0}^{v} f(s, u) d u-v f_{T}(s)\right] d s$. By Proposition 2 in the Appendix, under such alternatives, we have $L_{n}(t, 0.75)-L_{n}(0.25) \stackrel{\mathcal{D}}{\rightarrow}\left[\int_{0}^{t} y(s)^{-1}\left(G_{1}(d s, v)-v G_{1}(d s, 1)\right)\right]_{v=0.25}^{v=0.75}$, whose distribution can be arbitrarily close to the corresponding null distribution with the uniform marginal for $V$ as $\delta \rightarrow 0$. This example shows that the test based on $U_{3}$ under discretized marks can have nearly zero large-sample power for certain alternatives involving continuous marks. The same conclusion can be reached for $U_{1}$ and $U_{2}$.

We now show that the test statistics are asymptotically distribution-free when given the weight process $H_{n}(t)=\hat{S}_{C}(t-) \hat{S}_{T}^{1 / 2}(t)$ introduced in Section 2.3 . Let $\tilde{U}_{1}, \tilde{U}_{2}$, and $\tilde{U}_{3}$ denote the test statistics with this weight process. With $H(t)=S_{C}(t-) S_{T}^{1 / 2}(t)$, simple calculation shows that $\int_{0}^{t} H(s)^{2} / y(s)^{2} \gamma(d s, 1)=\int_{0}^{t} f_{T}(s) d s=F_{T}(t)$. If $\tilde{\tau}=\sup \left\{t: S_{T}(t)>0\right\}$, then, from the previous discussion and by the continuous mapping theorem, under $H_{0}$,

$$
\begin{aligned}
\tilde{U}_{1} \equiv & \sup _{v_{1}<v_{2}} \sup _{0 \leq t<\tilde{\tau}} \Delta\left(t, v_{1}, v_{2}\right) \stackrel{\mathcal{D}}{\rightarrow} \sup _{v_{1}<v_{2}} \sup _{0 \leq t<1} \Delta K\left(t, v_{1}, v_{2}\right) \\
\tilde{U}_{2} \equiv & \sup _{v_{1}<v_{2}} \sup _{0 \leq s \leq t<\tilde{\tau}}\left(\Delta\left(t, v_{1}, v_{2}\right)-\Delta\left(s, v_{1}, v_{2}\right)\right) \\
& \stackrel{\mathcal{D}}{\rightarrow} \sup _{v_{1}<v_{2}} \sup _{0 \leq s \leq t<1}\left(\Delta K\left(t, v_{1}, v_{2}\right)-\Delta K\left(s, v_{1}, v_{2}\right)\right) \\
\tilde{U}_{3} \equiv & \sup _{v_{1}<v_{2}} \sup _{0 \leq t<\tilde{\tau}}\left|L_{n}\left(t, v_{2}\right)-L_{n}\left(t, v_{1}\right)\right| \stackrel{\mathcal{D}}{\longrightarrow} \sup _{v_{1}<v_{2}} \sup _{0 \leq t<1}\left|K\left(t, v_{2}\right)-K\left(t, v_{1}\right)\right|,
\end{aligned}
$$

where $\Delta\left(t, v_{1}, v_{2}\right)$ is defined following (8), $\Delta K\left(t, v_{1}, v_{2}\right)=K\left(t, v_{1}\right)+K\left(t, v_{2}\right)-2 K\left(t,\left(v_{1}+v_{2}\right) / 2\right)$, and $K(t, v)$ is a Kiefer process with $\operatorname{Cov}(K(s, u), K(t, v))=(s \wedge t)(u \wedge v-u v)$. Therefore, the $\tilde{U}_{j}$ are asymptotically distribution-free test statistics. The asymptotic critical values of the tests $\tilde{U}_{j}$ can be tabulated through a single simulation study based on the known properties of the Kiefer process. This asymptotic distribution-free procedure, which applies only for the weight process $H_{n}(t)=\hat{S}_{C}(t-) \hat{S}_{T}^{1 / 2}(t)$, is computationally more efficient and provides a good alternative for large sample sizes. However, the proposed Monte Carlo procedure can be used for a broad class of weight processes, it provides more accurate critical values for moderate sample sizes, and it is not overly cumbersome computationally.

Remark 1. In some applications, it is of interest to evaluate whether the instantaneous or absolute risk of failure depends on a continuous mark variable in a given time interval, say $\left[t_{1}, t_{2}\right)$, rather than over the entire time range $[0, \tau)$. The null and alternative hypotheses, and the test statistics $U_{1}, U_{2}$, and $U_{3}$, can be modified straightforwardly to address this problem. All of the results given in this section carry over to this case, by replacing $[0, \tau)$ everywhere with $\left[t_{1}, t_{2}\right)$. In addition, the 
results continue to hold if the time range $[0, \tau)$ is replaced with the possibly larger range $[0, \tilde{\tau})$; see the remark following the proof of Theorem 2 in the Appendix.

\section{Simulation results}

We describe results of a simulation study of the test statistics $\tilde{U}_{1}, \tilde{U}_{2}$, and $\tilde{U}_{3}$.

First we consider a case with $T$ and $V$ independent. The cumulative incidence function is then $F(t, v)=P\{T \leq t\} f_{V}(v)$, where $f_{V}$ is the density of $V$. We specify $T$ to be exponential with mean 1 , and $f_{V}(v)=(1 / \beta) v^{(1 / \beta)-1}$ for $0 \leq v \leq 1$. Here $\beta=1.0$ corresponds to the null hypothesis $H_{0}$ and $\beta=0.75,0.5,0.25$ correspond to three different alternative hypotheses under the monotone alternatives $H_{1}$ and $H_{2}$. The extent of departure from the null hypothesis increases as $\beta$ decreases. We also consider a two-sided alternative with $f_{V}(v)=12(v-0.5)^{2}$, $0 \leq v \leq 1$ (results in this case are given under the heading "two-sided" in Tables 1 and 2). Next, we consider a case with $T$ and $V$ dependent. For the monotone alternatives $H_{1}$ and $H_{2}$, we use $F(t, v)=P\{T \leq t \mid V=v\} f_{V}(v)=(1-\exp (-t /(v+1))) f_{V}(v)$, with $f_{V}(v)=(1 / \beta) v^{(1 / \beta)-1}$ for $0 \leq v \leq 1$ and $\beta=0.5$ and 0.25 . For a two-sided alternative, we select $V$ from uniform $(0,1)$ and $F(t, v)=1-\exp \left(-v^{4} t\right)$.

We choose $n=50,100$ and use a $30 \%$ censoring rate for the failure times. The sizes and powers of the tests are calculated based on 1000 samples. The nominal level is set at 0.05 in each case. The critical level for each test is calculated using 1000 independent replicates of $\left\{V_{1}^{*}, \ldots, V_{n}^{*}\right\}$. The results in Table 1 indicate that the proposed tests perform well at moderate sample sizes. The estimated sizes are all within $1.5 \%$ of the nominal $5.0 \%$ (range: $3.9 \%$ to $6.5 \%$ ), and the estimated powers are high for detecting $\beta=0.25$ when $n=50$ (range: $81.3 \%$ to $100.0 \%$ ) and for detecting $\beta=0.50$ when $n=100$ (range: $72.0 \%$ to $86.6 \%$ ).

To this point, we have assumed that $T$ and $V$ are jointly continuous. In some applications, however, some ties may be present in the data. To study the sensitivity of the tests to the presence of ties, we use the same simulated data that yielded Table 1, and group the failure times into 25 tied values $x_{m}=0.05+0.1(m-1)$ for $0.1(m-1)<x \leq 0.1 m, m=1, \ldots, 24$ and $x_{25}=2.45$ for $x>2.4$ (or $x_{m}=3+6(m-1), m=1, \ldots, 24, x_{25}=147$ for the two-sided alternative model under dependent $T$ and $V$ ), and group the failure marks into 20 tied values $v_{m}=0.025+0.05(m-1)$ for $0.05(m-1)<v \leq 0.05 m, m=1, \ldots, 20$. As mentioned earlier, this testing procedure for the tied mark data is equivalent to the procedure of Sun (2001). The sizes and powers of the tests for the grouped data at a $30 \%$ censoring rate and the $5.0 \%$ nominal level are given in Table 
2. We note that the presence of ties causes a slight but consistent decrease in the power of the tests, and the levels become more conservative than in the untied case. The test based on $\tilde{U}_{3}$ is quite conservative for tied data, with estimated size $1.8 \%$ when $n=50$. The test becomes less conservative when the sample size is increased to $n=100$, with estimated size $3.3 \%$. An additional simulation (not reported here) shows that, as might be expected, the tests have decreasing power as the number of groups decreases.

The slightly larger sizes for $n=100$ compared with $n=50$ in Table 1 may be due to Monte Carlo error: The reported sizes are the proportion of rejections of $H_{0}$ in 1000 samples, so they have a Monte Carlo standard error of about $[0.05 * 0.95 / 1000]^{1 / 2} \times 100 \%=0.7 \%$.

\section{PLACE TABLES 1-2 HERE}

\section{Application}

In 1995 and 1996, the Adult AIDS Clinical Trials Group (AACTG) conducted a randomized trial (Study 241) of 400 HIV infected adults to evaluate two combination antiretroviral treatments by their ability to suppress HIV viral load (D'Aquila et al. 1996). The drug regimens contained zidovudine and didanosine plus either nevirapine or nevirapine placebo. Gilbert et al. (2000) analyzed the data from this trial with the failure time $T$ defined as the time from randomization until plasma HIV levels rose above 1000 copies/ml. The available genotypic data from the study are the amino acids at 19 codons in the reverse transcriptase of HIV isolated from peripheral blood mononuclear cells at baseline and at or after the time of failure from 12 patients on the dual-drug arm and 33 patients on the triple-drug arm who failed. The 19 codons were chosen on the basis of information from published studies that mutations in these positions confer resistance to at least one of the studied drugs (Gunthard et al. 1999; Leigh-Brown et al. 1999; Hanna et al. 2000). For the present analysis, codons with a resistance mutation are coded as ones while codons with nonresistant (whether wildtype or variant) or ambiguous amino acids are coded as zeros.

Let $V_{b}$ be the mutational distance of a subject's virus sequence measured at baseline relative to the "wildtype" virus with no mutations, defined by

$$
V_{b}=\sum_{i=1}^{19} w_{i} I(\text { mutation at codon } i) / \sum_{i=1}^{19} w_{i},
$$

where the weight $w_{i}$ measures the amount of resistance conferred by a mutation at the $i$ th position, as measured by a drug resistance assay. Define $V_{f}$ similarly for a subject's virus sequence measured 
at or after the time of failure (we refer to this time as the "late week"). Then, we take $V=V_{f}-\frac{2}{3} V_{b}$ as the measure of acquired mutational distance during the trial, which emphasizes new mutations more than baseline mutations. The weights $\left\{w_{i}\right\}$ are taken to be those used by Gilbert et al. (2000). Note that $V$ is only defined and measured on subjects who fail treatment, and therefore is appropriately viewed as a mark accompanying failure events rather than as a covariate. In the analysis we consider both treatment arms in a single group. Pooling the arms is meaningful because the accumulated resistance metric $V$ is relevant for both arms, as they share the nucleoside inhibitors zidovudine and didanosine.

\section{PLACE FIGURES 1-3 HERE}

As depicted in Figure 1, the mutational distance at baseline $V_{b}$ ranges between 0.0 and 0.187 in the 45 subjects who failed treatment, and increases to 0.0 to 0.435 by the late week, indicating a trend of increase in mutational distance during the trial. The observed mark variable $V$ takes 28 unique values for the 45 failures, ranging from 0.0 to 0.358 , and appears to be approximately uniformly distributed (Fig. 1c). A scatterplot of the mark versus failure time does not reveal a systematic pattern (Fig. 2). To implement the tests, we first normalize $V$ by its maximum observed value (0.358).

The tests confirm what is suspected from the descriptive plots, yielding nonsignificant results for the three alternative hypotheses, with test statistics $\tilde{U}_{1}=0.628(p=0.59), \tilde{U}_{2}=0.487$ $(p=0.64)$, and $\tilde{U}_{3}=0.353(p=0.76)$. We next implement the graphical procedure, which uses a unit weight process $H_{n}(t)=1$ in the test process $L_{n}(t, v)$ (as in (10)). When comparing the surface $L_{n}(t, v)$ to eight simulated surfaces $L_{n}^{*}(t, v)$ (Fig. 3), they appear similar except that $L_{n}(t, 0)$ rises above zero for increasing $t$ while the processes $L_{n}^{*}(t, 0)$ tend to remain closer to zero. Other than this caveat, which can be explained by the fact that four trial participants had a tied mark value $V=0$, the graphical comparison suggests that the observed test process does not behave unusually compared to the behavior expected under the null hypothesis. We conclude that there is no evidence that the instantaneous or absolute risk of virological failure depends on the level of the resistance mutational distance variable $V$ as defined above. Thus, $V$ may not be useful as a marker of drug resistance. It would be of interest to apply the testing procedure for several other metrics $V$, as an exploratory search for marks that indicate drug resistance.

\section{Concluding remarks}


The problem addressed here, evaluating whether there is a significant association between the instantaneous or absolute risk of failure and a continuous mark variable observed only at uncensored failure times, has broad application. The two cited applications in AIDS research, in which a time to disease or infection is measured and the mark describes a feature of the agent that causes or is associated with the failure event, arises in many biomedical applications. For one non-AIDS example, in studies evaluating survival of cancer patients, tumor mass might be measured in patients at baseline and at the time of death, and the tests can be used to evaluate a possible association between the growth rate of the tumor and the risk of death. In addition to many other biomedical applications, including the aforementioned problems of assessing the relationship between the risk of death and a quality of life score or a lifetime medical cost, there are a broad variety of applications in other scientific fields. Advantages of the tests developed here for addressing these problems include that they are based on a nonparametric maximum likelihood estimator which is a continuous generalization of the widely-applied and well-understood discrete cause-specific Nelson-Aalen estimator, and they are asymptotically distribution-free.

\section{Acknowledgments}

The authors thank the AACTG Study 241 Virology Team for providing the data for the example, namely George Hanna, Victor DeGruttola, Andrew Leigh Brown, Daniel Kuritzkes, Victoria Johnson, Douglas Richman, and Richard D'Aquila, and the anonymous referees for helpful comments.

\section{References}

E.-E. Aly, S.C. Kochar and I.W. McKeague, "Some tests for comparing cumulative incidence functions and cause-specific hazard rates," Journal of the American Statistical Association vol. 89 pp. 994-999, 1994.

P.K. Andersen, O. Borgan, N. Keiding and R. Gill, Statistical Models Based on Counting Processes, Springer: New York, 1993.

P.J. Bickel and M.J. Wichura, "Convergence criteria for multiparameter stochastic processes and some applications," Annals of Mathematical Statistics vol. 42 pp. 1656-1670, 1971.

Y. Bilias, M. Gu and Z. Ying, "Towards a general asymptotic theory for Cox model with staggered entry," Annals of Statistics vol. 25 pp. 662-682, 1997. 
P. Billingsley, Convergence of Probability Measures, Wiley: New York, 1968.

R.T. D’Aquila, M.D. Hughes, V.A. Johnson, M.A. Fischl, J.-P. Sommadossi, S. Liou, J. Timpone, M. Myers, N. Basgoz, M. Niu, M.S. Hirsch and the National Institute of Allergy and Infectious Diseases AIDS Clinical Trials Group Protocol 241 Investigators, "Nevirapine, zidovudine, and didanosine compared with zidovudine and didanosine in patients with HIV-1 infection," Annals of Internal Medicine vol. 124 pp. 1019-1031, 1996.

J.P. Fine, "Analysing competing risks data with transformation models," Journal of the Royal Statistical Society Series B vol. 61 pp. 817-830, 1999.

P.B. Gilbert, G.J. Hanna, V. De Gruttola, J. Martinez-Picado, D.R. Kuritzkes, V.A. Johnson, D.D. Richman and R.T. D'Aquila, "Comparative analysis of HIV type 1 genotypic resistance across antiretroviral trial treatment regimens," AIDS Research and Human Retroviruses vol. 16 pp. $1325-1336,2000$.

P.B. Gilbert, V. DeGruttola, S.M. Hammer and D.R. Kuritzkes, "Virological and regimen termination surrogate endpoints in AIDS clinical trials," Journal of the American Medical Association vol. 8 pp. $775-782,2001$.

P.B. Gilbert, S.G. Self, M. Rao, A. Naficy and J.D. Clemens, "Sieve analysis: Methods for assessing how vaccine efficacy depends on genotypic and phenotypic pathogen variation from vaccine trial data," Journal of Clinical Epidemiology vol. 54 pp. 68-85, 2001.

H.F. Gunthard, A.J. Leigh-Brown, R.T. D'Aquila, V.A. Johnson, D.R. Kuritzkes, D.D. Richman and J.K. Wong, "Higher selection pressure from antiretroviral drugs in vivo results in increased evolutionary resistance in HIV-1 pol," Virology vol. 259 pp. 154-165, 1999.

G.J. Hanna, V.A. Johnson, D.R. Kuritzkes, D.D. Richman, A.J. Leigh-Brown, A.V. Savara, J.D. Hazelwood and R.T. D'Aquila, "Patterns of resistance mutations selected by treatment of human immunodeficiency virus type 1 infection with zidovudine, didanosine, and nevirapine," The Journal of Infectious Diseases vol. 181 pp. 904-911, 2000.

M.S. Hirsch, F. Brun-Vezinet, R.T. D’Aquila, S.M. Hammer, V.A. Johnson, D.R. Kuritzkes, C. Loveday, J.W. Mellors, B. Clotet, B. Conway, L.M. Demeter, S. Vella, D.M. Jacobsen and D.D. Richman, "Antiretroviral drug resistance testing in adult HIV-1 infection: recommendations of an International AIDS Society-USA Panel," Journal of the American Medical Association 
vol. 283 pp. $2417-2426,2000$.

X.S. Hu and W.Y. Tsai, "Linear rank tests for competing risks model," Statistica Sinica vol. 9 pp. 971-983, 1999.

Y. Huang and T.A. Louis, "Nonparametric estimation of the joint distribution of survival time and mark variables," Biometrika vol. 85 pp. 785-798, 1998.

K.F. Lam, "A class of tests for the equality of $k$ cause-specific hazard rates in a competing risks model," Biometrika vol. 85 pp. 179-188, 1998.

A.J. Leigh-Brown, H.G. Gunthard, J.K. Wong, R.T. D’Aquila, V.A. Johnson, D.R. Kuritzkes and D.D. Richman, "Sequence clusters in human immunodeficiency virus type 1 reverse transcriptase are associated with subsequent virological response to antiretroviral therapy," The Journal of Infectious Diseases vol. 180 pp. 1043-1049, 1999.

X. Luo and B. Turnbull, "Comparing two treatments with multiple competing risks endpoints," Statistica Sinica vol. 9 pp. 985-997, 1999.

I.W. McKeague, A.M. Nikabadze and Y. Sun, "An omnibus test for independence of a survival time from a covariate," Annals of Statistics vol. 23 pp. 450-475, 1995.

I.W. McKeague and K.J. Utikal, "Identifying nonlinear covariate effects in semimartingale regression models," Probability Theory of Related Fields vol. 87 pp. 1-25, 1990.

M. Olschewski and M. Schumacher, "Statistical analysis of quality of life in cancer clinical trials," Statistics in Medicine vol. 9 pp. 749-763, 1990.

D. Pollard, Empirical Processes: Theory and Applications, NSF-CBMS Regional Conference Series in Probability and Statistics, 2. Institute of Mathematical Statistics, Hayward, CA, 1990.

R.L. Prentice, J.D. Kalbfleisch, A.V. Peterson, N. Flournoy, V.T. Farewell and N.E. Breslow, "The analysis of failure times in the presence of competing risks," Biometrics vol. 34 pp. $541-554,1978$.

G.R. Shorack and J.A. Wellner, Empirical Processes with Applications to Statistics, Wiley: New York, 1986.

Y. Sun, "Generalized nonparametric test procedures for comparing multiple cause-specific hazard 
rates," Journal of Nonparametric Statistics vol. 13 pp. 171-207, 2001.

Y. Sun and R.C. Tiwari, "Comparing cause-specific hazard rates of a competing risks model with censored data," IMS Lecture Notes-Monograph Series vol. 27 pp. 255-270, 1995. Analysis of Censored Data, edited by H. L. Koul and J. V. Deshpandé.

A.A. Tsiatis, "A nonidentifiability aspect of the problem of competing risks," Proceedings of the National Academy of Sciences USA vol. 72 pp. 20-22, 1975.

UNAIDS, Report from a Meeting of the WHO-UNAIDS Vaccine Advisory Committee, "Approaches to the development of broadly protective HIV vaccines: Challenges posed by genetic, biological and antigenic variability of HIV-1," AIDS vol. 15 pp. W1-W25, 2001.

A.W. Van der Vaart and J.A. Wellner, Weak Convergence and Empirical Processes with Applications to Statistics, Springer: New York, 1996.

P.G. Yeni, S.M. Hammer, C.C. Carpenter, D.A. Cooper, M.A. Fischl, J.M. Gatell, B. Gazzard, M.S. Hirsch, D.M. Jacobsen, D.A. Katzenstein, J.S. Montaner, D.D. Richman, M.S. Saag, M. Schechter, R.T. Schooley, M.A. Thompson, S. Vella and P.A. Volberding, "Antiretroviral treatment for adult HIV infection in 2002: updated recommendations of the International AIDS Society-USA Panel," Journal of the American Medical Association vol. 288 pp. 222$235,2002$.

\section{Appendix: Proofs of theorems}

The following lemma is needed in the proofs of the main results.

\section{Lemma}

Assume that $C$ is independent of $(T, V)$. Then

$$
\begin{aligned}
& \int_{0}^{t} \frac{\gamma(d s, v)}{y(s)}=\int_{0}^{t} \int_{0}^{v} \lambda(s, u) d u d s \\
& \int_{0}^{t} \frac{\gamma(d s, 1)}{y(s)}=\int_{0}^{t} \lambda(s) d s .
\end{aligned}
$$

Proof: Let $F_{C}(t)$ be the distribution function of the censoring variable $C$. Recalling the notation $f(t, v)$ for the joint density of $(T, V)$, we have

$$
\gamma(t, v)=P(T \leq t, T \leq C, V \leq v)=\int_{0}^{\infty} P(T \leq t, T \leq s, V \leq v) d F_{C}(s)
$$




$$
\begin{aligned}
& =\int_{0}^{\infty}\left(\int_{0}^{s \wedge t} \int_{0}^{v} f(r, u) d u d r\right) d F_{C}(s)=\int_{0}^{t} \int_{r}^{\infty} \int_{0}^{v} f(r, u) d u d F_{C}(s) d r \\
& =\int_{0}^{t} P(C \geq r) \int_{0}^{v} f(r, u) d u d r .
\end{aligned}
$$

It follows that

$$
\int_{0}^{t} \frac{\gamma(d s, v)}{y(s)}=\int_{0}^{t}\left(\int_{0}^{v} f(s, u) d u / P(T \geq s)\right) d s=\int_{0}^{t} \int_{0}^{v} \lambda(s, u) d u d s .
$$

This proves (A.1). The result (A.2) follows by letting $v=1$ and using $\int_{0}^{1} f(s, u) d u=f_{T}(s)$.

Proposition 1 describes the limiting distribution of the test process $L_{n}(t, v)$ when the weight function is unity.

\section{Proposition 1}

For $\tau<\tilde{\tau}$

$$
\begin{aligned}
& \sqrt{n}(\hat{\Lambda}(t, v)-\bar{\Lambda}(t, v))-\sqrt{n}\left(\Lambda(t, v)-v \int_{0}^{t} \lambda(s) d s\right) \\
& \stackrel{\mathcal{D}}{\longrightarrow} \int_{0}^{t} y(s)^{-1}\left(G_{1}(d s, v)-v G_{1}(d s, 1)\right)-\int_{0}^{t} G_{2}(s) y(s)^{2}(\gamma(d s, v)-v \gamma(d s, 1))
\end{aligned}
$$

in $D([0, \tau] \times[0,1])$.

Proof: By the empirical central limit theorem

$$
\sqrt{n}(N(t, v) / n-\gamma(t, v), Y(t) / n-y(t)) \stackrel{\mathcal{D}}{\rightarrow}\left(G_{1}(t, v), G_{2}(t)\right)
$$

in $D([0, \tau] \times[0,1]) \times D[0, \tau]$, where $G_{1}(t, v)$ and $G_{2}(t)$ are continuous mean zero Gaussian processes with covariances given in Theorem 1. Let $D=D([0, \tau] \times[0,1]) \times D[0, \tau]$ be the product space. The Nelson-Aalen-type estimator $\hat{\Lambda}(t, v)=\int_{0}^{t} N(d s, v) / Y(s), 0 \leq t \leq \tau$, depends on the pair $\left(n^{-1} N(t, v), n^{-1} Y(t)\right)$ through the following map from the domain of the type $D_{\phi}=$ $\left\{(A(t, v), B(t)): \int|A(d t, v)| \leq M, B(t) \geq \epsilon\right\} \subset D$ for given $M$ and $\epsilon>0$ :

$$
\phi:(A(t, v), B(t)) \rightarrow \int_{0}^{t} \frac{1}{B(s)} A(d s, v)
$$

Let $D_{0}=C([0, \tau] \times[0,1]) \times C[0, \tau]$. First, we show that the map $\phi$ is Hadamard-differentiable tangentially to the set $D_{0}$ at every point $(A, B)$ such that $1 / B(t)$ is of bounded variation. Let $t_{n} \rightarrow$ 0 be any converging sequences and let $\left(\alpha_{n}, \beta_{n}\right) \rightarrow(\alpha, \beta) \in D_{0}$ such that $\left(A+t_{n} \alpha_{n}, B+t_{n} \beta_{n}\right) \in D_{\phi}$. Then

$$
t_{n}^{-1}\left(\phi\left(A+t_{n} \alpha_{n}, B+t_{n} \beta_{n}\right)(t, v)-\phi(A, B)(t, v)\right)
$$




$$
\begin{aligned}
& =\int_{0}^{t} \frac{1}{B(s)+t_{n} \beta_{n}(s)} \alpha_{n}(d s, v)-\int_{0}^{t} \frac{\beta_{n}(s)}{B(s)\left(B(s)+t_{n} \beta_{n}(s)\right)} A(d s, v) \\
& \rightarrow \int_{0}^{t} \frac{1}{B(s)} \alpha(d s, v)-\int_{0}^{t} \frac{\beta(s)}{B(s)^{2}} A(d s, v), \text { uniformly in }(t, v) \\
& =\phi_{A, B}^{\prime}(\alpha, \beta)(t, v),
\end{aligned}
$$

where the limit is obtained by applying a lemma of Bilias, Gu, and Ying (1997). Let

$$
Z_{n}(t, v)=\sqrt{n}(\hat{\Lambda}(t, v)-\Lambda(t, v))
$$

Since the pair $\left(n^{-1} N(t, v), n^{-1} Y(t)\right),(t, v) \in[0, \tau] \times[0,1]$, is contained in the domain $D_{\phi}$ with probability tending to 1 for $M \geq 1$ and sufficiently small $\epsilon>0$, applying the functional delta method theorem (van der Vaart and Wellner, 1996, p. 374), (A.1) and (A.5), we have

$$
Z_{n}(t, v) \stackrel{\mathcal{D}}{\rightarrow} Z(t, v)
$$

where

$$
Z(t, v)=\int_{0}^{t} \frac{1}{y(s)} G_{1}(d s, v)-\int_{0}^{t} \frac{G_{2}(s)}{y(s)^{2}} \gamma(d s, v) .
$$

Now, consider the following continuous map from $D([0, \tau] \times[0,1])$ into itself,

$$
\psi_{1}: \quad \psi_{1}(g)(t, v)=g(t, v)-v g(t, 1), \quad(t, v) \in[0, \tau] \times[0,1] .
$$

Applying the continuous mapping theorem, we get

$$
Z_{n}(t, v)-v Z_{n}(t, 1) \stackrel{\mathcal{D}}{\rightarrow} Z(t, v)-v Z(t, 1), .
$$

Proposition 1 follows by plugging the specific forms (A.6) and (A.8) of the processes $Z_{n}$ and $Z$ into (A.10) .

Proposition 2 extends the result of Proposition 1 to general weight processes.

\section{Proposition 2}

Given the conditions expressed in Theorem 1,

$$
\begin{aligned}
& L_{n}(t, v)-\sqrt{n}\left(\int_{0}^{t} \int_{0}^{v} H_{n}(s) \lambda(s, u) d u d s-v \int_{0}^{t} H_{n}(s) \lambda(s) d s\right) \\
& \stackrel{\mathcal{D}}{\longrightarrow} \int_{0}^{t} H(s) y(s)^{-1}\left(G_{1}(d s, v)-v G_{1}(d s, 1)\right)-\int_{0}^{t} H(s) G_{2}(s) y(s)^{2}(\gamma(d s, v)-v \gamma(d s, 1))
\end{aligned}
$$

in $D([0, \tau] \times[0,1])$. 
Proof: By the almost sure representations theorem (Shorack and Wellner 1986, p. 47), there exist $\tilde{N}(t, v), \tilde{Y}(t), \tilde{G}_{1}(t, v)$ and $\tilde{G}_{2}(t)$ on some probability space such that $\tilde{N}(t, v), \tilde{Y}(t), \tilde{G}_{1}(t, v)$ and $\tilde{G}_{2}(t)$ are equal in law to $N(t, v), Y(t), G_{1}(t, v)$ and $G_{2}(t)$, respectively, and (A.4) holds almost surely uniformly in $(t, v)$. Furthermore $\tilde{N}(t, v), \tilde{Y}(t), \tilde{G}_{1}(t, v)$ and $\tilde{G}_{2}(t)$ can be chosen to have the same sample paths as the original processes. Let $\tilde{Z}_{n}$ and $\tilde{Z}$ be the corresponding representations of $Z_{n}$ and $Z$ defined in (A.6) and (A.8), respectively. Repeating the steps of (A.5) with $t_{n}=n^{-1 / 2}, \alpha_{n}(t, v)=\sqrt{n}(N(t, v) / n-\gamma(t, v))$ and $\beta_{n}(t)=\sqrt{n}(Y(t) / n-y(t))$ and applying the lemma of Bilias, Gu, and Ying (1997), we have

$$
\tilde{Z}_{n}(t, v) \stackrel{\text { a.s. }}{\longrightarrow} \tilde{Z}(t, v) \text {, uniformly in }(t, v) \text {. }
$$

Consequently,

$$
\tilde{Z}_{n}(t, v)-v \tilde{Z}_{n}(t, 1) \stackrel{\text { a.s. }}{\rightarrow} \tilde{Z}(t, v)-v \tilde{Z}(t, 1) .
$$

Let $\tilde{H}_{n}(t)$ be the process $H_{n}(t)$ redefined in terms of $\tilde{N}(t, v)$ and $\tilde{Y}(t)$. Then $\tilde{H}_{n}(t)$ has the properties of $H_{n}(t)$ assumed in the theorem. Applying the lemma of Bilias, Gu, and Ying (1997), we have

$$
\int_{0}^{t} \tilde{H}_{n}(s)\left(\tilde{Z}_{n}(d s, v)-v \tilde{Z}_{n}(d s, 1)\right) \stackrel{\text { a.s. }}{\longrightarrow} \int_{0}^{t} H(s)(\tilde{Z}(d s, v)-v \tilde{Z}(d s, 1)) .
$$

Hence

$$
\int_{0}^{t} H_{n}(s)\left(Z_{n}(d s, v)-v Z_{n}(d s, 1)\right) \stackrel{\mathcal{D}}{\longrightarrow} \int_{0}^{t} H(s)(Z(d s, v)-v Z(d s, 1)),
$$

in $D([0, \tau] \times[0,1])$. Proposition 2 follows by plugging the specific forms (A.6) and (A.8) of the processes $Z_{n}$ and $Z$ into (A.12) and by (5).

Proof of Theorem 1: Under $H_{0}$, the failure time $T$ and the failure mark $V$ are independent and $V$ is uniformly distributed on $[0,1]$. Consequently, $\lambda(t, v)=\lambda(t)$ for all $(t, v)$. Further, since the censoring variable $C$ is independent of $(T, V), V$ is independent of $(T, C)$ under $H_{0}$. Hence, $\gamma(t, v)=v \gamma(t, 1)$ under $H_{0}$. The result (11) follows by applying Proposition 2 .

Let $N_{i}(t)=I\left(X_{i} \leq t, \delta_{i}=1\right)$. By (A.4), under $H_{0}$ we have

$$
\left.n^{-1 / 2} \sum_{i=1}^{n} N_{i}(t)\left(I\left(V_{i} \leq v\right)-v\right)\right) \stackrel{\mathcal{D}}{\rightarrow} G_{1}(t, v)-v G_{1}(t, 1)
$$

in $D([0, \tau] \times[0,1])$. Since $H(t)$ and $1 / y(t)$ are of bounded variation over $t \in[0, \tau]$, the map $\psi_{2}: g(t, v) \rightarrow \int_{0}^{t} H(s) y(s)^{-1} g(d s, v),(t, v) \in[0, \tau] \times[0,1]$ from $D([0, \tau] \times[0,1])$ into itself is continuous on $C([0, \tau] \times[0,1])$. Hence, by the continuous mapping theorem,

$$
\left.n^{-1 / 2} \sum_{i=1}^{n}\left(I\left(V_{i} \leq v\right)-v\right)\right) \int_{0}^{t} \frac{H(s)}{y(s)} N_{i}(d s) \stackrel{\mathcal{D}}{\rightarrow} \int_{0}^{t} \frac{H(s)}{y(s)}\left(G_{1}(d s, v)-v G_{1}(d s, 1)\right)=L(t, v) .
$$


The covariance of $L(t, v)$ is given by, under the independence between $V$ and $(X, \delta)$,

$$
\begin{aligned}
\operatorname{Cov}(L(s, u), L(t, v)) & \left.\left.=E\left\{\left(I\left(V_{i} \leq u\right)-u\right)\right)\left(I\left(V_{i} \leq v\right)-v\right)\right)\right\} E\left\{\int_{0}^{s} \frac{H(r)}{y(r)} N_{i}(d r) \int_{0}^{t} \frac{H(r)}{y(r)} N_{i}(d r)\right\} \\
& =(u \wedge v-u v) \int_{0}^{s \wedge t} \frac{H^{2}(r)}{y(r)^{2}} \gamma(d r, 1) .
\end{aligned}
$$

Remark 2. An alternative (and more elegant) proof of Theorem 1 could be developed using empirical process techniques. This would involve an extension to $\hat{\Lambda}$ of Pollard's (1990, Section 13) derivation of the limiting distribution of the Nelson-Aalen estimator.

Proof of Theorem 2: $\quad$ Let $L_{1}^{*}(t, v)=\sqrt{n}\left(\hat{\Lambda}^{*}(t, v)-v \hat{\Lambda}^{*}(t, 1)\right)$. Then $L_{n}^{*}(t, v)=\int_{0}^{t} H_{n}(s) L_{1}^{*}(d s, v)$. Let $L_{1}(t, v)=\int_{0}^{t} y(s)^{-1}\left(G_{1}(d s, v)-v G_{1}(d s, 1)\right)$. It is sufficient to show $L_{1}^{*} \stackrel{\mathcal{D}}{\rightarrow} L_{1}$ conditionally in $D([0, \tau] \times[0,1])$.

Let $N_{i}(t)=I\left(X_{i} \leq t, \delta_{i}=1\right)$ and $N(t)=\sum_{i=1}^{n} N_{i}(t)$. Then

$$
L_{1}^{*}(t, v)=\sqrt{n} \sum_{i=1}^{n}\left(I\left(V_{i}^{*} \leq v\right)-v\right) \int_{0}^{t} \frac{d N_{i}(s)}{Y(s)} .
$$

To establish the conditional weak convergence of $L_{1}^{*}(t, v)$, we shall show that the finite dimensional distributions of $L_{1}^{*}(t, v)$ converge weakly to those of $L_{1}(t, v)$ given the data sequence, and that $L_{1}^{*}(t, v)$ is asymptotically tight given the data sequence; see van der Vaart and Wellner (1996, p. 183). The former task can be done by a simple application of the central limit theorem. The asymptotic tightness of $L_{1}^{*}(t, v)$ given the data sequence can be proved by applying the tightness criteria of Bickel and Wichura (1971, eq. 3, p. 1658) based on neighboring blocks. The details are contained in a technical report that can be requested from the authors.

\section{Proof of Theorem 3:}

(a) Let

$$
m(t, v)=\int_{0}^{t} \int_{0}^{v} H_{n}(s) \lambda(s, u) d u d s-v \int_{0}^{t} H_{n}(s) \lambda(s) d s .
$$

Denote the left and the right side of (A.11) by $L_{n}^{a}(t, v)$ and $L^{a}(t, v)$, respectively. Then

$$
\begin{aligned}
& U_{1}= \sup _{v_{1}<v_{2}} \sup _{0 \leq t<\tau} \Delta\left(t, v_{1}, v_{2}\right) \\
&= \sup _{v_{1}<v_{2}} \sup _{0 \leq t<\tau}\left[\left(L_{n}^{a}\left(t, v_{1}\right)+L_{n}^{a}\left(t, v_{2}\right)-2 L_{n}^{a}\left(t,\left(v_{1}+v_{2}\right) / 2\right)\right)\right. \\
&\left.\quad+\sqrt{n}\left(m\left(t, v_{1}\right)+m\left(t, v_{2}\right)-2 m\left(t,\left(v_{1}+v_{2}\right) / 2\right)\right)\right] \\
& \geq \sqrt{n} \sup _{v_{1}<v_{2}} \sup _{0 \leq t<\tau}\left(\left(m\left(t, v_{1}\right)+m\left(t, v_{2}\right)-2 m\left(t,\left(v_{1}+v_{2}\right) / 2\right)\right)\right) \\
& \text { estion of } \quad-\sup _{v_{1}<v_{2}} \sup _{0 \leq t<\tau}\left[-\left(L_{n}^{a}\left(t, v_{1}\right)+L_{n}^{a}\left(t, v_{2}\right)-2 L_{n}^{a}\left(t,\left(v_{1}+v_{2}\right) / 2\right)\right)\right]
\end{aligned}
$$


Note that

$$
\begin{aligned}
& m\left(t, v_{1}\right)+m\left(t, v_{2}\right)-2 m\left(t,\left(v_{1}+v_{2}\right) / 2\right) \\
\stackrel{\text { a.s. }}{\longrightarrow} \int_{0}^{t} H(s)\left(\int_{0}^{v_{1}} \lambda(s, u) d u+\int_{0}^{v_{2}} \lambda(s, u) d u-2 \int_{0}^{\left(v_{1}+v_{2}\right) / 2} \lambda(s, u) d u\right) d s & \int_{0}^{t}\left[H(s) / S_{T}(s)\right] d\left(\int_{0}^{v_{1}} F(s, u) d u+\int_{0}^{v_{2}} F(s, u) d u-2 \int_{0}^{\left(v_{1}+v_{2}\right) / 2} F(s, u) d u\right) \\
= & {\left[H(t) / S_{T}(t)\right]\left(\int_{0}^{v_{1}} F(t, u) d u+\int_{0}^{v_{2}} F(t, u) d u-2 \int_{0}^{\left(v_{1}+v_{2}\right) / 2} F(t, u) d u\right) } \\
& \quad-\int_{0}^{t}\left(\int_{0}^{v_{1}} F(s, u) d u+\int_{0}^{v_{2}} F(s, u) d u-2 \int_{0}^{\left(v_{1}+v_{2}\right) / 2} F(s, u) d u\right) d\left(H(s) / S_{T}(s)\right) \\
\geq & {\left[H(t) / S_{T}(t)\right]\left(\int_{0}^{v_{1}} F(t, u) d u+\int_{0}^{v_{2}} F(t, u) d u-2 \int_{0}^{\left(v_{1}+v_{2}\right) / 2} F(t, u) d u\right), }
\end{aligned}
$$

where the last inequality is obtained since $\int_{0}^{v_{1}} F(s, u) d u+\int_{0}^{v_{2}} F(s, u) d u-2 \int_{0}^{\left(v_{1}+v_{2}\right) / 2} F(s, u) d u$ $\geq 0$ under $H_{1}$ and $H(s) / S_{T}(s)$ is decreasing. Under $H_{1}$ with the given $t_{0}$ such that the inequality of the alternative $H_{1}$ holds strictly for some $\left(v_{1}, v_{2}\right) \in[0,1], \int_{0}^{v} F\left(t_{0}, u\right) d u$ is a strictly concave function. Hence

$$
\int_{0}^{v_{1}} F\left(t_{0}, u\right) d u+\int_{0}^{v_{2}} F\left(t_{0}, u\right) d u-2 \int_{0}^{\left(v_{1}+v_{2}\right) / 2} F\left(t_{0}, u\right) d u>0
$$

for some $v_{1}, v_{2} \in[0,1]$. By Proposition 2, the second term of (A.14) converges in distribution to a finite random variable. This yields $U_{1} \stackrel{P}{\rightarrow} \infty$. Since $U_{1}^{*}$ converges in distribution to a finite random variable, $U_{1}$ is consistent against $H_{1}$.

(b) Note that, for $s<t$,

$$
\begin{aligned}
& m\left(t, v_{1}\right)+m\left(t, v_{2}\right)-2 m\left(t,\left(v_{1}+v_{2}\right) / 2\right)-m\left(s, v_{1}\right)+m\left(s, v_{2}\right)-2 m\left(s,\left(v_{1}+v_{2}\right) / 2\right) \\
& \stackrel{\text { a.s. }}{\longrightarrow} \int_{s}^{t} H(r)\left(\int_{0}^{v_{1}} \lambda(r, u) d u+\int_{0}^{v_{2}} \lambda(r, u) d u-2 \int_{0}^{\left(v_{1}+v_{2}\right) / 2} \lambda(r, u) d u\right) d r .
\end{aligned}
$$

Under $H_{2}$, with the given $t_{0}$ such that the inequality of the alternative $H_{2}$ holds strictly for some $\left(v_{1}, v_{2}\right) \in[0,1], \int_{0}^{v} \lambda\left(t_{0}, u\right) d u$ is a strictly concave function. Hence

$$
\int_{0}^{v_{1}} \lambda\left(t_{0}, u\right) d u+\int_{0}^{v_{2}} \lambda\left(t_{0}, u\right) d u-2 \int_{0}^{\left(v_{1}+v_{2}\right) / 2} \lambda\left(t_{0}, u\right) d u>0
$$

for some $v_{1}, v_{2} \in[0,1]$. Following a similar argument as in part (a) of the proof for the consistency of $U_{1}$, applying Proposition 2, and by the continuity assumptions of the theorem, we have $U_{2} \stackrel{P}{\rightarrow} \infty$. Since $U_{2}^{*}$ converges in distribution to a finite random variable, $U_{2}$ is consistent against $\mathrm{H}_{2}$. 
(c) Note that the alternative $H_{3}$ that $\lambda(t, v)$ does not depend on $v$ for all $t \in[0, \tau]$ is equivalent to $\int_{0}^{t} H(s)\left[\int_{0}^{v}(\lambda(s, u)-\lambda(s)) d u\right] d s$ not depending on $v$ for all $t \in[0, \tau]$. Thus, under $H_{3}$ there exists $\left(t_{0}, v_{1}, v_{2}\right), t_{0} \in[0, \tau],\left(v_{1}, v_{2}\right) \in[0,1]$ such that

$$
m\left(t_{0}, v_{2}\right)-m\left(t_{0}, v_{1}\right) \stackrel{\text { a.s. }}{\rightarrow} \int_{0}^{t_{0}} H(s)\left[\int_{v_{1}}^{v_{2}}(\lambda(s, u)-\lambda(s)) d u\right] d s \neq 0 .
$$

Since

$$
\begin{aligned}
U_{3} \geq & \sqrt{n} \sup _{v_{1}<v_{2}} \sup _{0 \leq t<\tau}\left|m\left(t, v_{2}\right)-m\left(t, v_{1}\right)\right| \\
& \quad-\sup _{v_{1}<v_{2}} \sup _{0 \leq t<\tau}\left|\left(L_{n}\left(t, v_{2}\right)-\sqrt{n} m\left(t, v_{2}\right)\right)-\left(L_{n}\left(t, v_{1}\right)-\sqrt{n} m\left(t, v_{1}\right)\right)\right|,
\end{aligned}
$$

it follows by Proposition 2, the continuous mapping theorem and (A.15) that $U_{3} \stackrel{P}{\rightarrow} \infty$ under $H_{3}$. Since $U_{3}^{*}$ converges in distribution to a finite random variable, $U_{3}$ is consistent against $H_{3}$. 
Table 1. Observed levels and powers (\%) of the test statistics $\tilde{U}_{1}, \tilde{U}_{2}, \tilde{U}_{3}$ for testing $H_{0}$ versus $H_{1}, H_{2}, H_{3}$, respectively, at the $5.0 \%$ nominal level.

\begin{tabular}{c|c|ccccc|ccc}
\hline \hline & & \multicolumn{6}{|c|}{ Independent $T$ and $V$} & \multicolumn{3}{|c}{ Dependent $T$ and $V$} \\
\hline \multirow{3}{*}{ Size $n$} & & \multicolumn{6}{|c|}{$\beta$} & \multicolumn{4}{|c}{$\beta$} \\
\hline \multirow{5}{*}{50} & Test & 1 & 0.75 & 0.5 & 0.25 & two-sided & 0.5 & 0.25 & two-sided \\
\hline \multirow{5}{*}{100} & $\tilde{U}_{1}$ & 5.9 & 21.1 & 55.2 & 88.2 & 19.2 & 49.5 & 85.0 & 17.9 \\
& $\tilde{U}_{2}$ & 5.5 & 17.5 & 48.6 & 83.1 & 14.9 & 46.0 & 81.3 & 12.1 \\
& $\tilde{U}_{3}$ & 3.9 & 12.5 & 60.0 & 100.0 & 99.0 & 55.8 & 99.9 & 56.3 \\
\hline \multirow{3}{*}{10} & $\tilde{U}_{1}$ & 6.5 & 27.7 & 78.2 & 99.2 & 62.1 & 80.2 & 98.9 & 29.4 \\
& $\tilde{U}_{2}$ & 6.0 & 23.9 & 72.0 & 98.4 & 54.3 & 76.0 & 98.0 & 19.7 \\
& $\tilde{U}_{3}$ & 5.7 & 20.5 & 84.5 & 100.0 & 100.0 & 86.6 & 100.0 & 85.0 \\
\hline
\end{tabular}


Table 2. Observed levels and powers (\%) of the test statistics $\tilde{U}_{1}, \tilde{U}_{2}, \tilde{U}_{3}$ for testing $H_{0}$ versus $H_{1}, H_{2}, H_{3}$, respectively, with tied data at the $5.0 \%$ nominal level.

\begin{tabular}{c|c|ccccc|ccc}
\hline \hline & & \multicolumn{6}{|c|}{ Independent $T$ and $V$} & \multicolumn{3}{|c}{ Dependent $T$ and $V$} \\
\hline \multirow{3}{*}{ Size $n$} & & \multicolumn{6}{|c|}{$\beta$} & \multicolumn{4}{|c}{$\beta$} \\
\hline \multirow{5}{*}{50} & Test & 1 & 0.75 & 0.5 & 0.25 & two-sided & 0.5 & 0.25 & two-sided \\
\hline \multirow{5}{*}{100} & $\tilde{U}_{1}$ & 4.5 & 16.8 & 50.3 & 85.7 & 17.4 & 40.5 & 82.1 & 9.8 \\
& $\tilde{U}_{2}$ & 4.2 & 13.9 & 41.7 & 77.3 & 11.8 & 36.2 & 74.5 & 3.0 \\
& $\tilde{U}_{3}$ & 1.8 & 8.6 & 52.4 & 99.8 & 96.6 & 39.4 & 99.7 & 42.0 \\
\hline \multirow{3}{*}{10} & $\tilde{U}_{1}$ & 4.0 & 24.9 & 74.0 & 99.0 & 55.3 & 71.1 & 97.3 & 25.0 \\
& $\tilde{U}_{2}$ & 4.0 & 19.6 & 65.2 & 97.7 & 45.7 & 64.6 & 95.8 & 11.0 \\
& $\tilde{U}_{3}$ & 3.3 & 13.5 & 79.1 & 100.0 & 100.0 & 78.3 & 100.0 & 86.3 \\
\hline
\end{tabular}




\section{FIGURE CAPTIONS}

Figure 1. Genetic data from the 45 trial participants in AACTG Study 241 who failed antiretroviral therapy. The frequency distribution of the mutational distance is shown (i) at baseline $\left(V_{b}\right)$, (ii) at the late week $\left(V_{f}\right)$, and (iii) accumulated between baseline and the late week $\left(V=V_{f}-\frac{2}{3} V_{b}\right)$. The mark $V$ is the scaled weighted sum of indicators of zidovudine or didanosine resistance mutations at positions $41,65,67,69,70,74,210,215$, and 219 in the reverse transcriptase gene, and of the nevirapine resistance mutations at positions $98,100,101,103,106,108,179,181,188$, and 190 in the reverse transcriptase gene. Details about the types of mutations, including the selected weights based on the level of in vitro drug susceptibility, can be found in Gilbert et al. (2000).

Figure 2. Accumulated mutational distance $V$ versus failure time, for the 45 subjects in clinical trial AACTG Study 241 who failed antiretroviral therapy. The line in the plot is a lowess curve that smooths the data in windows that contain two-thirds of the nearest data-points.

Figure 3. For clinical trial AACTG Study 241, for the testing procedures that account for ties in the mark variable, (a) plots the test process $L_{n}(t, v)$; (b)-(i) plot individual realizations of the simulated test processes $L_{n}^{*}(t, v)$. 
(i) mutational distance at baseline

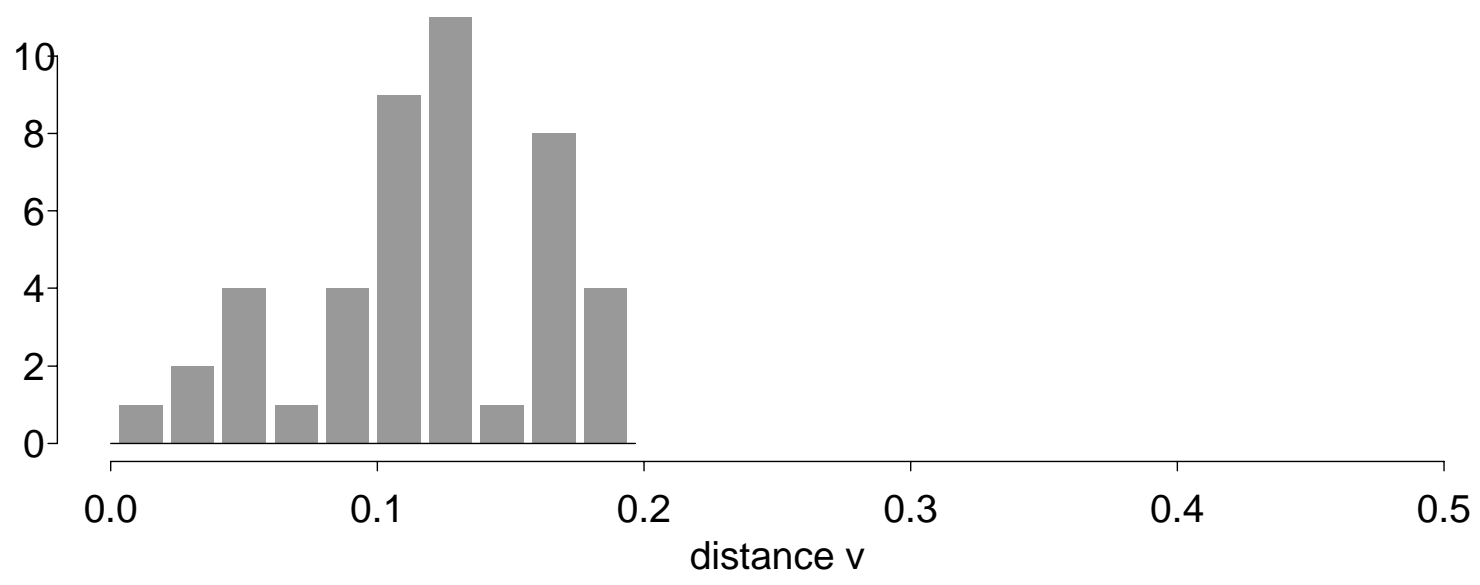

(ii) mutational distance at the late week

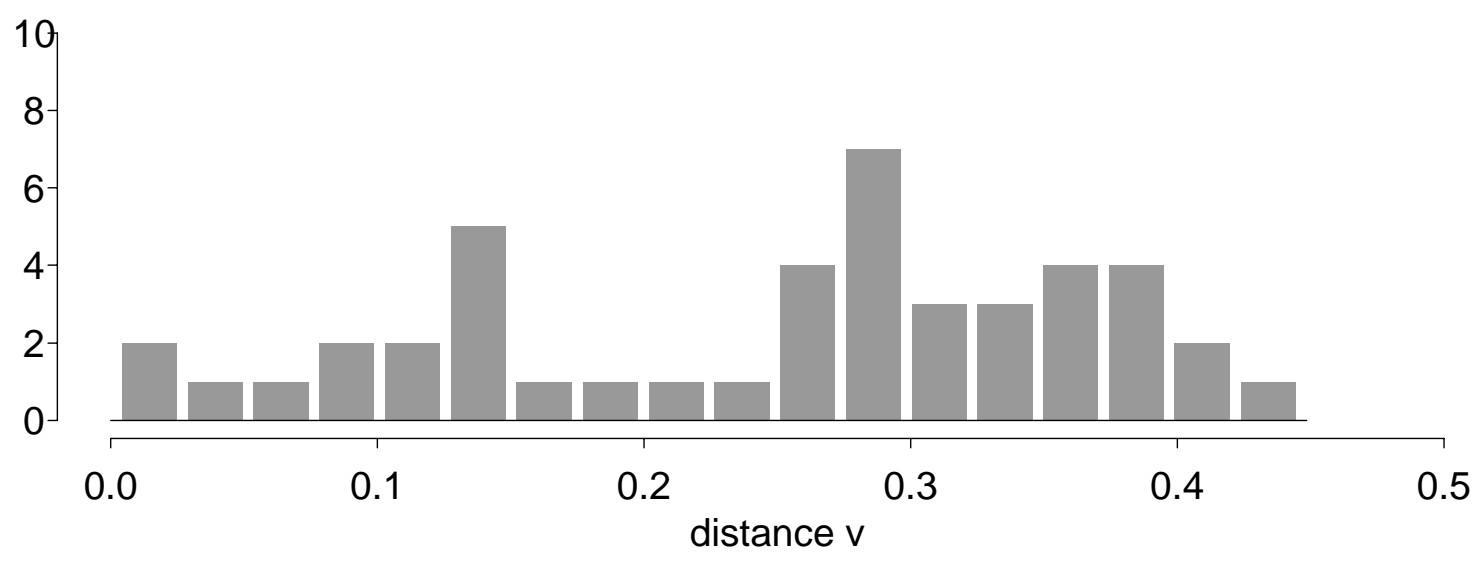

(iii) mutational distance $\mathrm{V}$ accumulated during the trial

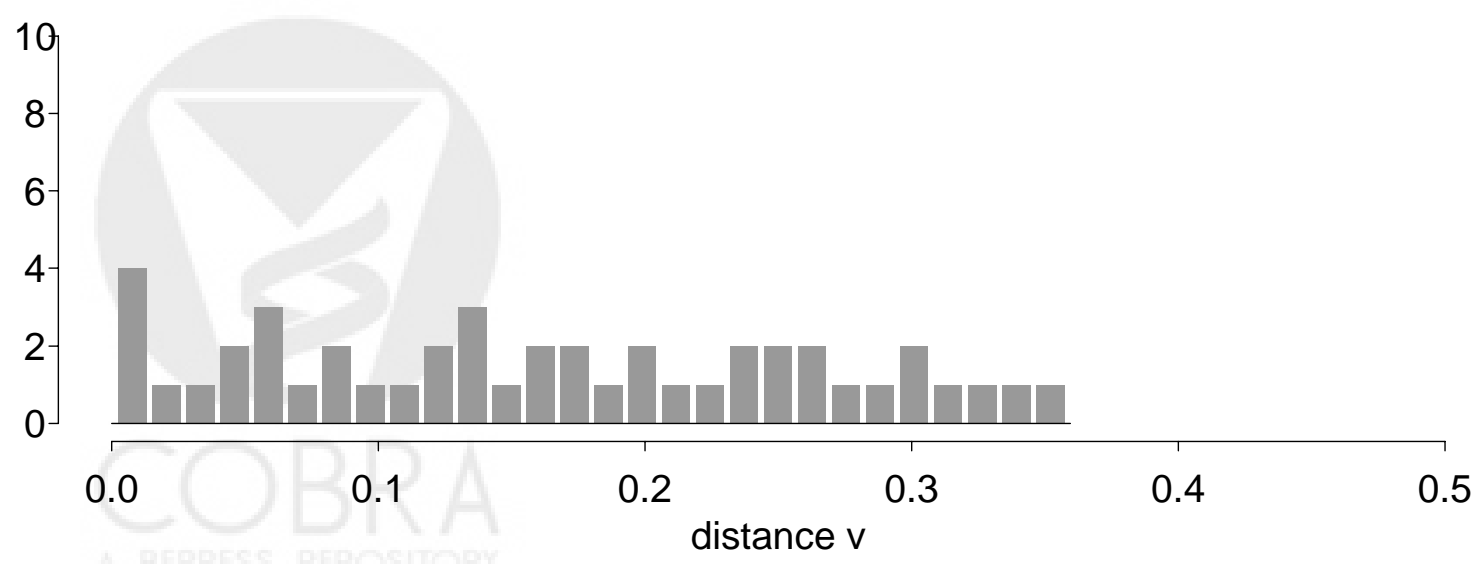




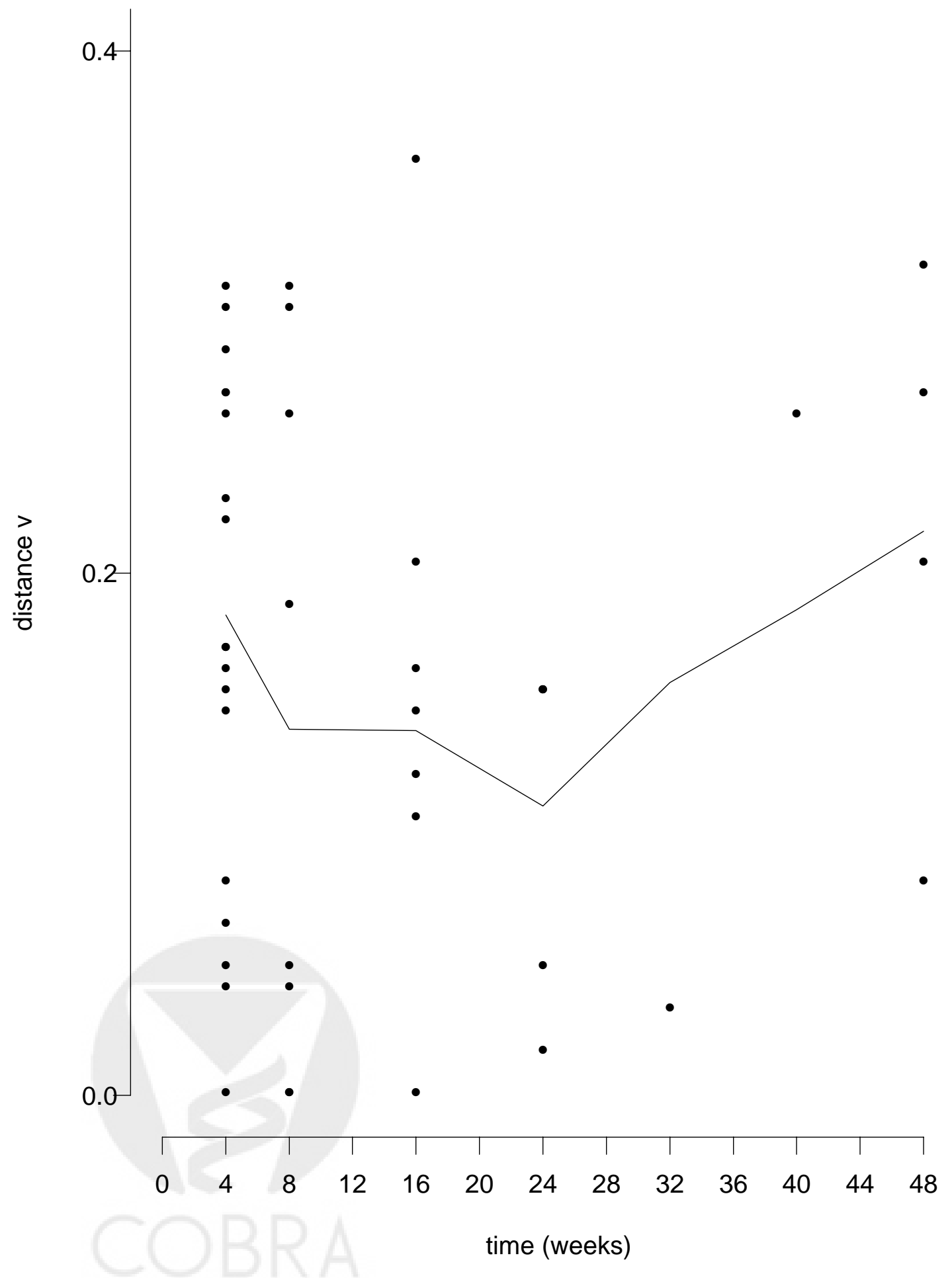




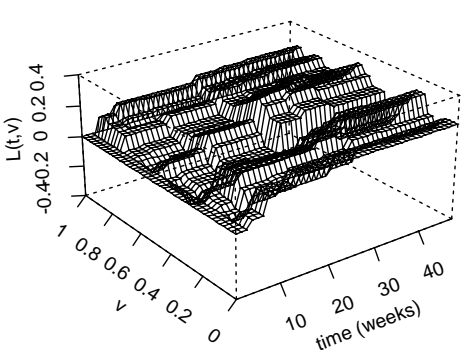

(a)

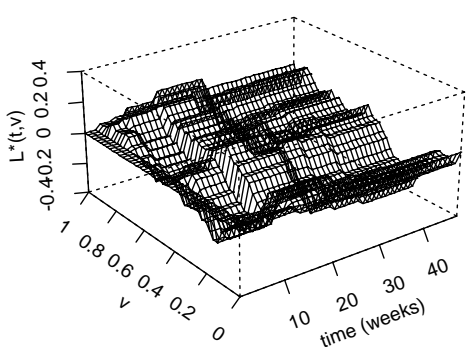

(d)

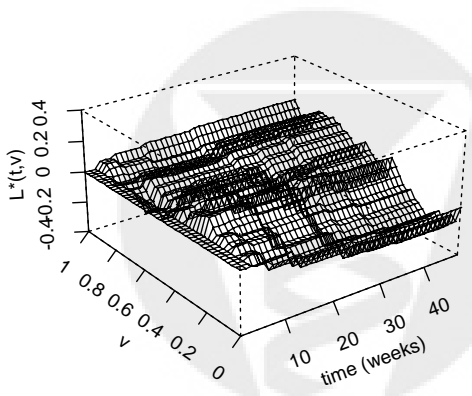

(g)

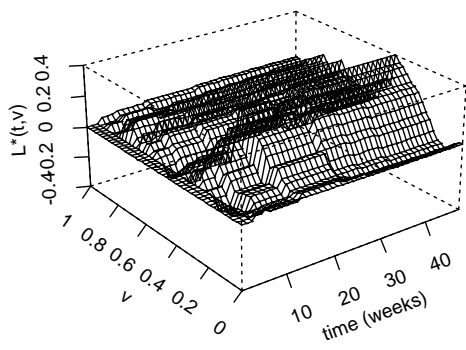

(b)

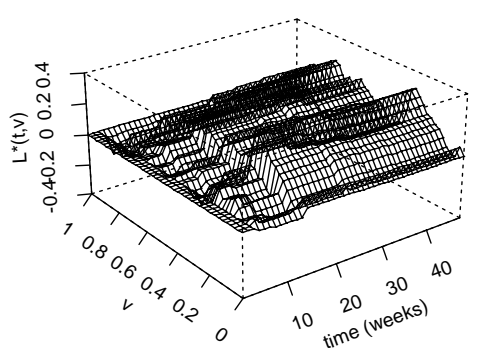

(e)

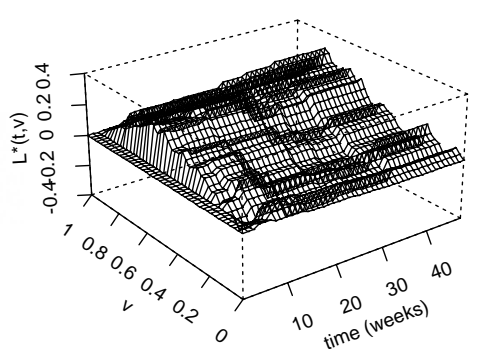

(h)

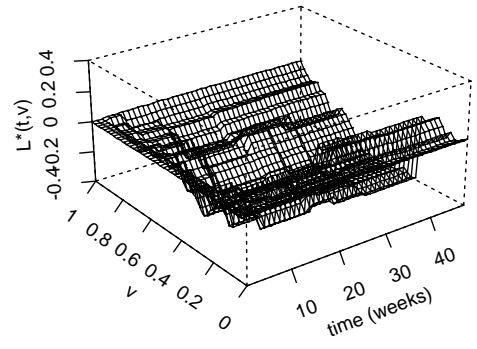

(c)

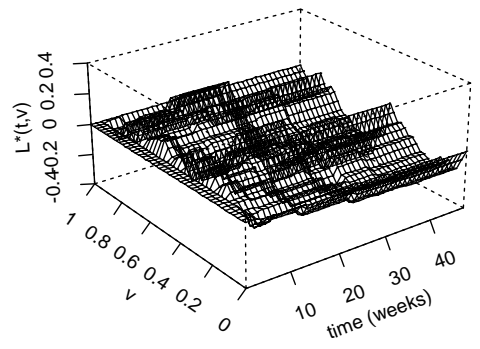

(f)

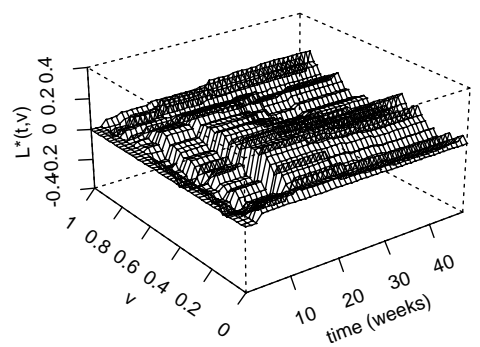

(i) 